\title{
Genotypic prevalence of norovirus Gll in gastroenteritis outpatients in Shanghai from 2016 to 2018
}

\author{
Xiaozhou Kuang ${ }^{\dagger}$, Zheng Teng ${ }^{\dagger}$ and Xi Zhang ${ }^{*}$
}

\begin{abstract}
Background: With the help of an existing citywide comprehensive surveillance on gastroenteritis outpatients, although norovirus genogroup II (NoV GII) was tested routinely, its genotypes were never investigated systematically on a municipal level. This study aimed to understand the prevalence, major genotypes and evolutional trends of NoV GII in Shanghai during the period of 2016-2018, and to provide molecular bases for early warning for any potential NoV outbreaks.
\end{abstract}

Methods: 27 sentinel hospitals from all 16 districts were recruited by stratified probability proportional to size (PPS) method in Shanghai comprehensive diarrhea surveillance programme. Stool samples were collected and screened for NoV GIl by real-time reverse transcription polymerase chain reaction (qRT-PCR). For samples that were positive in qRTPCR, conventional RT-PCR was performed to amplify the ORF1-ORF2 junction of NoV Gll gene. Generated sequences were typed by RIVM online genotyping tool, and then strains of interest were analyzed phylogenetically using MEGA 6.0.

Results: A total of 7883 stool samples were collected from diarrhea outpatients, among which 6474 were from adults and 1409 were from children. 13.66\% (1077 cases) were screened positive in qRT-PCR for NoV Gll, from which 71.96\% (775 cases) were sequenced successfully. The top three genotypes were GII.Pe/GII.4 (37\%), GII.P17/GIl.17 (26\%) and GII.P16/GII.2 (17\%). While GII.Pe/GIl.4 detection rate decreased significantly over the 3 years (from 48.4 to 20.9\%); Gll. P16/Gll.2 appeared for the first time in October 2016 and rose rapidly to 27.0\% in 2017, but fell back to 23.4\% in 2018. Meanwhile there was a significant increase for both GII.P12/GIl.3 and GII.P7/GIl.6 recombinant genotypes detected in adult population in 2018. Phylogenic analysis revealed the existence of multiple gene clusters within both of these recombinant genotypes.

Conclusion: Unlike the alternating circulation of Gll.4 and non-GIl.4 NoV observed in 2016 or 2017, the genotype profile of NoV Gll in 2018 was characterized by the co-prevalence of multiple recombinant genotypes. A recent increase in detection rate in less reported recombinant genotypes such as GII.P12/GII.3 and GII.P7/GIl.6 among adult population calls for a continuing close monitoring on NoV Gll genotypes in case of potential local outbreaks.

Keywords: Norovirus GII, Gastroenteritis outpatients, Genotype, Recombinant strain

\footnotetext{
*Correspondence: zhangxi@scdc.sh.cn

${ }^{+}$Xiaozhou Kuang and Zheng Teng contributed equally to this work

Shanghai Municipal Center for Disease Control and Prevention, 1380

Zhongshan Road (west), Shanghai 200336, China
} 


\section{Background}

Norovirus (NoV) is the leading cause of acute gastroenteritis worldwide. All age groups are susceptible to its infection, and its detection rate ranks first in both sporadic cases and outbreaks [1-3]. The economic burden attributed to NoV infection on the health care system is about $\$ 4.2$ billion, and the burden on social economy is as high as $\$ 60.3$ billion [4]. Previous comprehensive monitoring of gastroenteritis outpatients in Shanghai had revealed that $\mathrm{NoV}$ was found in $\sim 60 \%$ of all diarrhea outpatients [5], among which NoV GII accounted for $90 \%$ of all $\mathrm{NoV}$ infections [6].

In terms of its molecular evolution, NoV is susceptible to rapid mutations through gene recombination, which is commonly observed in its ORF1-ORF2 junction [7]. In China, GII.Pe/GII.4 Sydney strain first appeared in 2012 [8], then a new GII.17 strain was found during the winter of 2014 [9], subsequently a recombinant GII.P16/GII.2 genotype emerged at the end of 2016 [10]. In Shanghai, GII.Pe/GII.4 Sydney strain was first reported In September 2012 [11], but its dominance was gradually replaced by GII.P17/GII.17 in 2015 [12]. Up until 2016, the circulation of local NoV genotypes was similar to that reported nationwide, and around the world [13-15]. This study aimed to ascertain the time of first appearance of local GII.P16/GII.2 strain after 2016, and to describe the prevalence and circulation of other genotypes in the past 3 years including GII.P12/GII.3 and GII.P7/GII.6, which were increasingly reported recently worldwide in outbreaks [16-18]. Finding in this study could provide scientific basis for the early warning of potential NoV GII outbreaks.

\section{Methods}

\section{Case definition}

Outpatients who sought medical care at the sentinel clinics, with a daily bowel movements 3 times or more, accompanied by loose or liquid stools [the definition of diarrhea by the World Health Organization (WHO)] [19].

\section{Sentinel site recruitment and case sampling}

Sentinel recruitment for comprehensive surveillance of diarrheal diseases programme in Shanghai were detailed in previous literature [6]: briefly, all hospitals containing enteric clinics in Shanghai were divided into urban and suburban groups, and then each group was further stratified into primary, secondary and tertiary hospital subgroups, and finally 27 hospitals are drawn by this stratified PPS method, covering 22 adults and 5 pediatric sentinel sites. Adult enteric clinics recruited outpatients over the age of 14, while pediatric clinics recruited outpatients under 14-year according to the definition of these two types of clinics in China. Diarrhea cases were sampled at pre-estimated sampling intervals in each sentinel clinic.

\section{Specimen collection and storage}

$5 \mathrm{~g}$ of stool was collected into a plastic cup without the addition of any chemical substances on the day of patient's hospital visit. It was then temporarily stored at $4{ }^{\circ} \mathrm{C}$ at the sentinel site and delivered in an ice-packed biosafety transport carrier to the laboratory on the same day.

\section{Sample process prior to RNA Extraction}

$5 \%$ saline solution was added to the sample to make a $10 \%$ fecal suspensions, it was then thoroughly vortexed, aliquoted and then centrifuged at $\times 8000 \mathrm{~g}$ for $5 \mathrm{~min}$ for immediate testing.

\section{RNA extraction}

$200 \mu \mathrm{l}$ of the centrifuged supernatant was aspirated, and extracted using Roche MagNA Pure LC 2.0 extractor with Roche Total Nucleic Acid Isolation Kit (Roche Applied Science, Switzerland) according to the manufacturer's instructions.

\section{qRT-PCR screening}

All specimens were double screened for NoV GII by qRTPCR using commercially available kits (Shanghai Zhijiang Biotechnology Co., Ltd. and Jiangsu Shuoshi Biotechnology Co., Ltd.). Both kits targeted the ORF1-ORF2 junction of NoV GII gene.

\section{NoV GIl genotyping}

For samples that were positive in both qRT-PCR kits, NoV GII ORF1-ORF2 junction (also known as the polymerase-capsid region) was amplified using method designed by the US CDC [20]. QIAxcel capillary electrophoresis was run with QIAxcel DNA Screening Kit (Qiagen, Hilden, Germany) to determine whether the specimen was successfully amplified and showed an expectant product size of $570 \mathrm{bp}$. All specimens positive with the target fragment size were sequenced by $\mathrm{ABI}$ sequencer 3730 DNA analyzer with BigDye ${ }^{\mathrm{TM}}$ Terminator v3.1 kit (Applied Biosystems, California, US). The resulting sequences were spliced using Sequncher software v4.1.4 (Gene Codes, US) [21] and then genotyped by RIVM online Norovirus genotyping tool (http://www. rivm.nl/mpf/norovirus/typingtool,RIVM,MA Bilthoven, Netherlands). Sequences representative of the main variants of the recombinant strains focused in this study were deposited in GenBank (accession numbers MK779279MK779304; MK789447-MK789463-Additional file data) (Additional file 1). 


\section{Phylogenic analysis}

For strains of interest, phylogenetic trees were constructed using the neighbour-joining method in MEGA version 6.0 software. Sequences were aligned using Clustal W with the Kimura-2 parameter. Tree robustness was determined by bootstrapping using 1000 pseudo replicates [22].

\section{Statistical analysis}

All calculations were conducted using Microsoft Excel 2010 and SPSS software v16.0 (IBM, USA) where Pearson Chi square test or the Fisher's exact with two-tailed method was used to determine statistical significance with $\mathrm{P}<0.05$.

\section{Results}

\section{NoV GII positive detection rate}

From January 2016 to December 2018, a total of 7883 stool samples were collected from diarrhea outpatients, including 2896 samples from 2016, 2622 samples in 2017, and 2365 samples from 2018. A total of 1077 samples (13.66\%) were positive for NoV GII by qRTPCR (Table 1). NoV GII screen positive rate in adults was significantly higher than that in children $\left(X^{2}=8.725\right.$, $\mathrm{P}<0.05)$.

\section{Seasonal variation in NoV Gll detection rate}

The positive detection rate generally peaked from autumn/winter to the next spring. It reached a historical apex at 37.93\% in the winter of 2017 (Fig. 1). On the contrary, when the average atmospheric temperature was highest in the summers, NoV GII detection rate plummeted as shown in Fig. 1.

\section{NoV ORF1-ORF2 (polymerase-capsid region) genotypes overall constitution}

Of 1077 NoV GII qRT-PCR positive samples, 775 were successfully sequenced. A total of 16 genotypes were obtained, of which, five of the most prominent genotypes were GII.Pe/GII.4, GII.P17/GII.17, GII.P16/GII.2, GII.P12/GII.3 and GII.P7/GII.6. Among these, 71.61\% (555 samples) were recombinant genotypes (Table 2). No

Table 1 NoV GII qRT-PCR screening results in adult and children outpatients in 2016-2018

\begin{tabular}{|c|c|c|c|c|c|c|c|c|}
\hline \multirow[t]{2}{*}{ Year } & \multicolumn{4}{|l|}{ Adults } & \multicolumn{4}{|l|}{ Children } \\
\hline & No. of cases & Screen POS no. & $\begin{array}{l}\text { Screen POS } \\
\text { rate (\%) }\end{array}$ & $x^{2}$ (P-value) & No. of cases & Screen POS no. & $\begin{array}{l}\text { Screen POS } \\
\text { rate (\%) }\end{array}$ & $\mathrm{X}^{2}$ (P-value) \\
\hline 2016 & 2443 & 339 & 13.88 & $18.02(<0.05)$ & 453 & 50 & 11.04 & $0.58(=0.75)$ \\
\hline 2017 & 2123 & 352 & 16.58 & & 499 & 60 & 12.02 & \\
\hline 2018 & 1908 & 228 & 11.95 & & 457 & 48 & 10.50 & \\
\hline Total & 6474 & 919 & 14.20 & & 1409 & 158 & 11.21 & \\
\hline
\end{tabular}

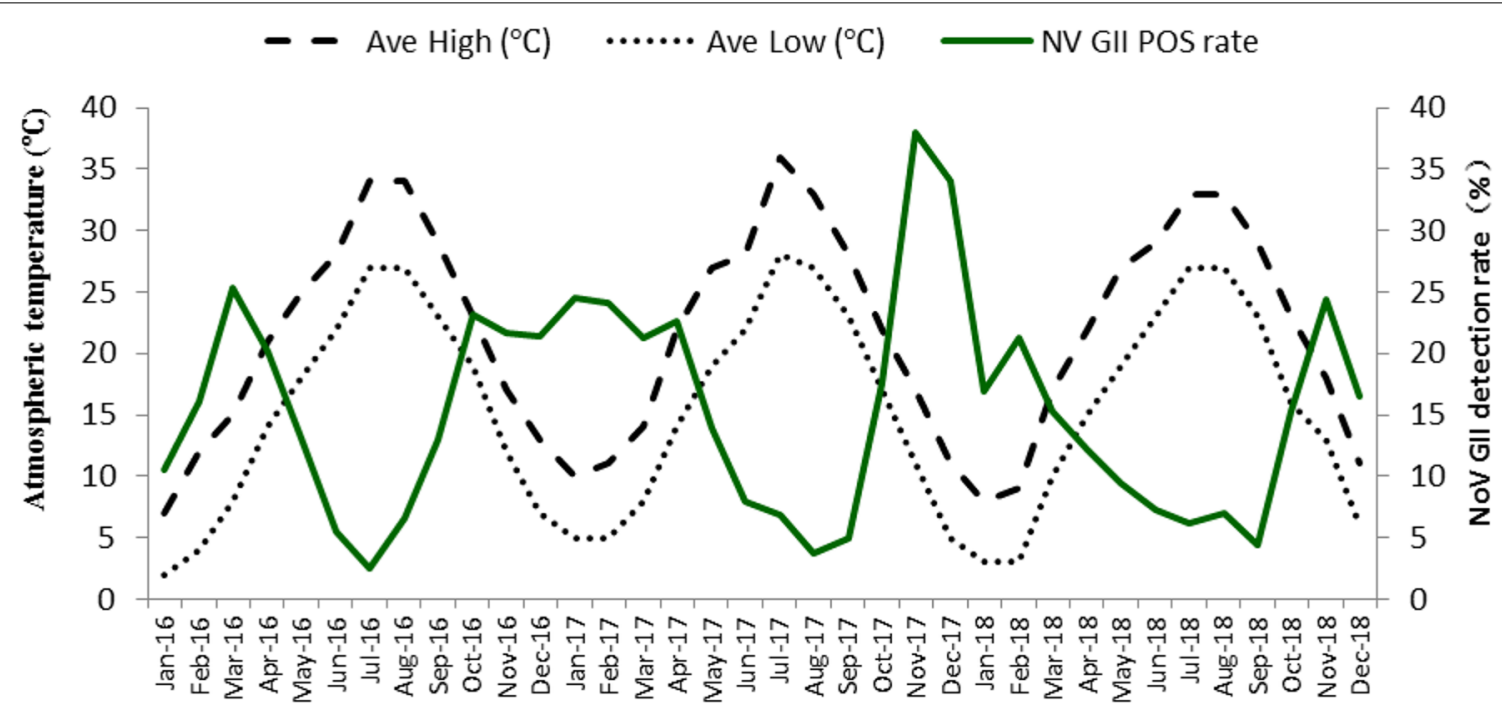

Fig. 1 Changes in the positive detection rate of NoV Gll at different times and atmospheric temperatures in Shanghai during $2016-2018$ 
Table 2 Compositional percentage of NoV GII genotypes found in 2016-2018

\begin{tabular}{lcl}
\hline $\begin{array}{l}\text { Polymerase-capsid region } \\
\text { genotype }\end{array}$ & No. of samples & $\begin{array}{l}\text { Compositional } \\
\text { percentage (\%) }\end{array}$ \\
\hline GII.Pe/GII.4 & 288 & 37.16 \\
GII.P17/GII.17 & 198 & 25.55 \\
GII.P16/GII.2 & 131 & 16.90 \\
GII.P12/GII.3 & 53 & 6.84 \\
GII.P7/GII.6 & 27 & 3.48 \\
GII.P16/GII.13 & 23 & 2.97 \\
GII.P21/GII.21 & 11 & 1.42 \\
GII.Pe/GII.17 & 10 & 1.29 \\
GII.P16/GII.4 & 8 & 1.03 \\
GII.P8/GII.8 & 6 & 0.77 \\
GII.P21/GII.13 & 6 & 0.77 \\
GII.P15/GII.15 & 5 & 0.65 \\
GII.P22/GII.5 & 4 & 0.52 \\
GII.Pg/GII.1 & 3 & 0.39 \\
GII.P7/GII.14 & 1 & 0.13 \\
GII.P7/GII.9 & 1 & 0.13 \\
Total & 775 & 100 \\
\hline
\end{tabular}

co-infection by different NoV GII genotypes was discovered in these samples.

\section{Time distribution of main genotypes}

For the predominant GII.Pe/GII.4 genotype, its proportion in January and August-to-November of 2016 as well as in October-to-December of 2017 exceeded the total proportion of all other genotypes at that time (Fig. 2). Moreover the prevalence of GII.Pe/GII.4 declined over
3 years (Table 3). Meanwhile although the second most prominent genotype GII.P17/GII.17 was prevalent throughout the year and remained relatively stable over the whole period, its percentage dropped to the lowest in August-to-October of 2016 and winter in 2017. It is worth noting that GII.P16/GII.2, which ranked third in prevalence, first appeared in October 2016 and continued to rise in 2017 and become the dominant genotype along with GII.Pe/GII.4 and GII.P17/GII.17. On the other hand, by 2018, the proportion of GII.P12/GII.3 and GII. P7/GII.6 had gradually increased, and the peak of GII. P7/GII.6 appeared around the summer of 2018, while the peak of GII.P12/GII.3 appeared in October-December 2018 (Table 3, Fig. 2).

\section{Comparison of NoV Gll genotypes found in adults and children outpatients}

658 sequences were obtained from adult cases, which can be further divided into 16 genotypes. The five most prevalent types were GII.Pe/GII.4 (214 cases, 32.52\%), GII. P17/GII.17 (194 cases, 29.48\%), GII.P16/GII.2 (110 cases, 16.72\%), GII.P12/GII.3 (41 cases, 6.23\%), GII.P7/GII.6 (24 cases, $3.65 \%$ ) (Table 4). The remaining genotypes include GII.P16/GII.13 (23 cases, 3.50\%), GII.Pe/GII.17 and GII. P21/GII.21 (10 cases each, 1.52\%), GII.P8/GII. 8, GII.P16/ GII.4 and GII.P21/GII.13 (6 cases each, 0.91\%), GII.P15/ GII.15 (5 cases, 0.76\%), GII.P22/GII.5 (4 cases, 0.61\%), GII.Pg/GII.1 (3 cases, 0.46\%), GII.P7/GII.14 and GII.P7/ GII.9 (1 case each, $0.15 \%$ ).

Meanwhile, 117 sequences were obtained from children's cases, which can be further divided into 7 genotypes. GII.Pe/GII.4 accounted for the highest proportion (74 cases, 63.25\%), it is followed by GII.P16/GII.2 (21

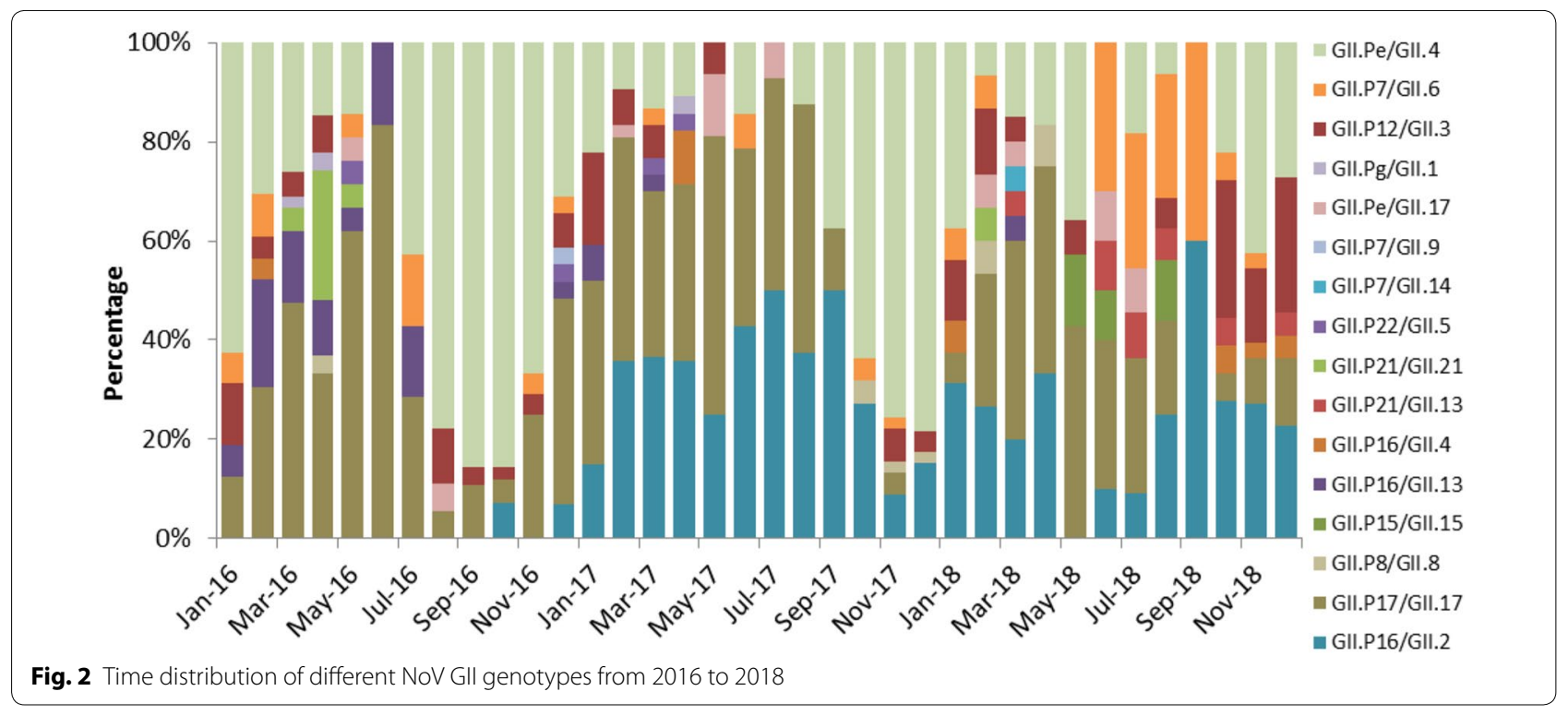


Table 3 Annual change in compositional percentage of main genotypes

\begin{tabular}{|c|c|c|c|c|c|}
\hline \multirow[t]{2}{*}{ Genotype } & \multicolumn{5}{|c|}{ Number/Percentage (\%) } \\
\hline & 2016 & 2017 & 2018 & Total & $\mathrm{X}^{2}$ (P-value) \\
\hline Gll.Pe/Gll.4 & 137 (48.41\%) & 107 (35.67\%) & $44(22.92 \%)$ & $288(37.16 \%)$ & $32.31(<0.05)$ \\
\hline Gll.P17/GIl.17 & $82(28.98 \%)$ & $76(25.33 \%)$ & $40(20.83 \%)$ & $198(25.55 \%)$ & $4.00(=0.14)$ \\
\hline Gll.P16/GIl.2 & $5(1.77 \%)$ & $81(27.00 \%)$ & $45(23.44 \%)$ & $131(16.90 \%)$ & $73.77(<0.05)$ \\
\hline GII.P12/GIl.3 & $14(4.95 \%)$ & $16(5.33 \%)$ & $23(11.98 \%)$ & $53(6.84 \%)$ & $10.62(<0.05)$ \\
\hline GII.P7/GIl.6 & $7(2.47 \%)$ & $4(1.33 \%)$ & $16(8.33 \%)$ & $27(3.48 \%)$ & $18.41(<0.05)$ \\
\hline Others & $38(13.43 \%)$ & $16(5.33 \%)$ & $24(12.5 \%)$ & $78(10.06 \%)$ & $12.21(<0.05)$ \\
\hline Total & $283(36.52 \%)$ & $300(38.71 \%)$ & 192 (24.77\%) & $775(100.00 \%)$ & \\
\hline
\end{tabular}

cases, 17.95\%), GII.P12/GII.3 (12 cases, 10.26\%), GII.P17/ GII.17 (4 cases, 3.42\%), GII.P7/GII.6 (3 cases, 2.56\%), GII. P16/GII.4 (2 cases, 1.71\%) and GII.P21/GII.21 (1 case, $0.85 \%$ ) Table 4 summarized the above finding.

The percentage of GII.Pe/GII.4 in both adults and children declined significantly over the years, and its prevalence in children was higher than that in adults. GII. P16/GII.2 underwent a significant change over 3 years in both adults and children, but no overall difference in its prevalence was observed between adults and children. The prevalence of GII.P17/GII.17 stayed relatively stable in both populations throughout the period, but its prevalence in adults was higher than that in children. While the prevalence of GII.P12/GII.3 and GII.P7/GII.6 in children remained stable over 3 years, significant changes was detected in adults for both strains (Table 4). Figures 3 and 4 shows the time distribution of all genotypes in both populations.

\section{Partial genetic analysis of less reported genotypes GII. P12/GII.3 and GII.P7/GII.6 that displayed a significant rise recently in adults}

NoV GII.P12/GII.3 phylogenic analysis showed that its polymerase region is basically divided into two clusters, one group clustered with reference strains reported in China from 2009 to 2017 and in Korea from 2006. Among these, strains isolated in this study from 2016 accounted for $11.9 \%$ (5/42), strains isolated from 2017 accounted for $33.3 \%(14 / 42)$ and strains isolated from 2018 accounted for $54.8 \%$ (23/42) of all. A second group clustered with reference strain found in Zhengzhou in 2017. Among these, local strains isolated from 2016 occupied 88.9\% (8/9) and strain isolated from 2017 occupied 11.1\% (1/9). Additional, there were two lone strains which belong to neither of these two clusters (Fig. 5a).

Phylogenic tree of partial ORF2 gene of GII.P12/GII.3 is also divided into two clusters. One group clustered with 2017 Zhengzhou reference strain and other reference strains reported in China from 2014 to 2015. Among these, local strain isolated from 2016 accounted for
28.3\%. (13/46), strains isolated from 2017 accounted for $23.9 \%(11 / 46)$ and strains isolated from 2018 accounted for $47.8 \%(22 / 46)$ of all. A second group clustered with Taizhou 2016-2017 reference strain. Among these, local strains isolated from 2017 occupied 83.3\% (5/6), strains isolated from 2018 occupied 16.7\% (1/6). There was only one lone strain which cannot be classified into either of two clusters (Fig. 5b).

NoV GII.P7/GII.6 phylogenic analysis showed that its polymerase region is basically divided into two clusters, one group clustered with reference strains reported from China during 2015-2016, from Vietnam during 20092010, from 2014 US and 2016 UK. Among these, strains isolated in this study from 2016 accounted for $43.75 \%$ (7/16), strains isolated from 2017 accounted for $18.75 \%$ $(3 / 16)$ and strains isolated from 2018 accounted for $37.5 \%$ (6/16) of all. A second group clustered with reference strain found in 2010 Brazil, 2011 Uruguay, 2011 Russia, 2013 Japan and 2016 China. Among these, local strains isolated from 2017 occupied 9.1\% (1/11) and strains isolated from 2018 occupied 90.9\% (10/11) (Fig. 6a).

Phylogenic tree of partial ORF2 gene of GII.P7/GII.6 is also divided into two clusters. One group clustered with 2010 Brazil, 2011 Uruguay, 2011 Russia, 2013 Japan and 2016 China. Among these, local strain isolated from 2017 accounted for $9.09 \%$ (1/11) and strains isolated from 2018 accounted for $90.91 \%(10 / 11)$ of all. A second group clustered with reference strains reported from China during 2015-2016, from Vietnam during 2009-2010, from 2014 US and 2016 UK. Among these, local strains isolated from 2016 occupied 43.75\% (7/16), strains isolated from 2017 occupied 18.75\% (3/16), strains isolated from 2018 occupied 37.50\% (6/16) (Fig. 6b).

\section{Discussion \\ NoV detection rate}

The average detection rate of NoV GII (13.66\%) was slightly higher than $10.43 \%$ reported in the diarrhea outpatients surveillance carried out in 27 provinces in 2009-2013 [23]; it was also higher than 10.50\% 


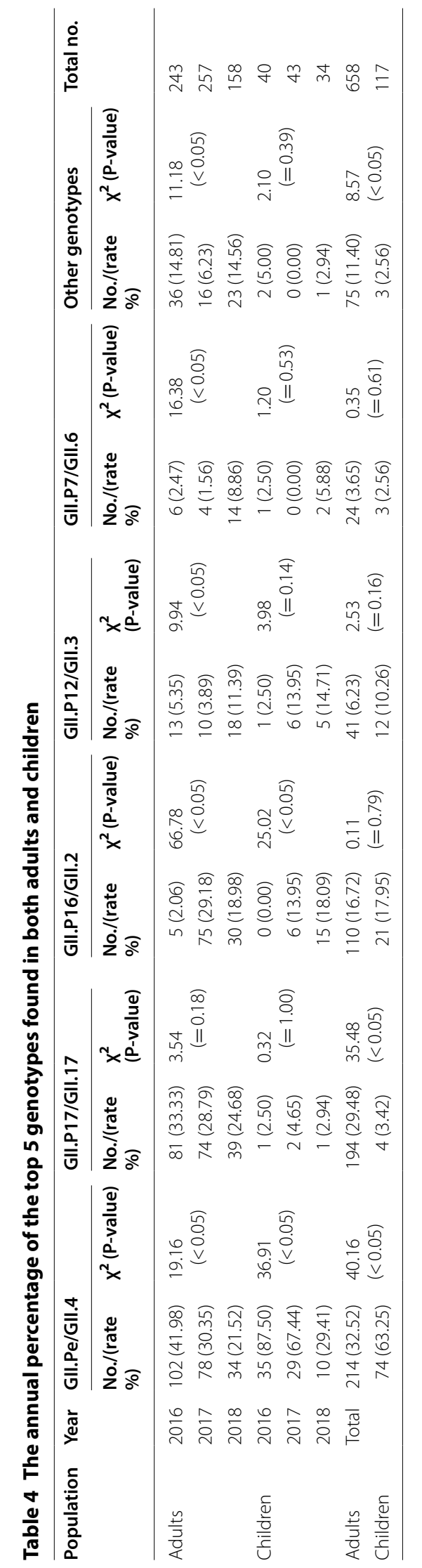




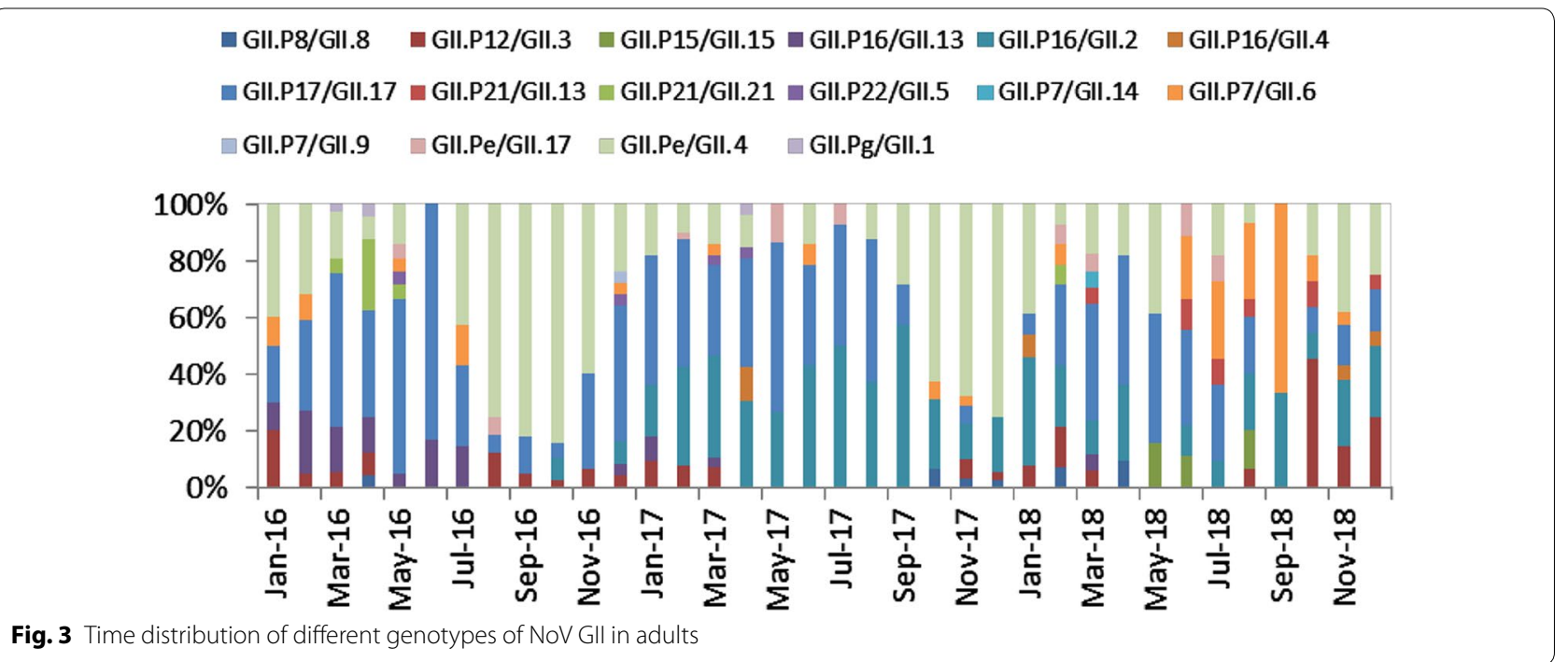

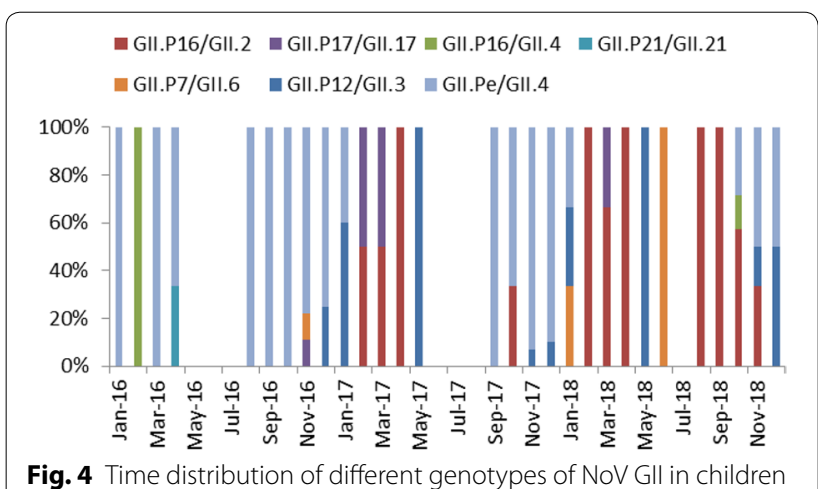

described in study in southwestern province during 2014-2015 [24]. As only conventional RT-PCR was used in these researches, a higher $\mathrm{NoV}$ detection rate in this study might be attributed to a more sensitive qRT-PCR method. Although NoV GII detection rate in children was comparable to that in equivalent population in the neighboring city of Nanjing during 2017-2018 [25], it is significantly lower than that in adults. This might be caused by the large difference in sampling size between two populations.

\section{Seasonal changes in NoV GIl infection rate}

The seasonal characteristics of local NoV GII were similar to that depicted in other provinces in China [23, $24]$, with its peak detection rate appeared in autumn/ winter to early spring, and its lowest detection rate in summer. A 10-year study in Hong Kong suggested that high atmospheric temperatures were statistically associated with low hospital admission rates caused by $\mathrm{NoV}$ infection, and that high relative humidity was also associated with high hospital admission rates [26]. Since this study lacks information on the temperature and relative humidity at the onset of each case, it is not possible to verify the Hong Kong study from a statistical point of view, but the general changing pattern of NoV positive rate over temperature change seems to be consistent with the finding in Hong Kong.

Evidence from other studies has demonstrated that with the increase in temperature, norovirus lost its viability and infectivity rapidly $[27,28]$. Therefore NoV seems to be more stable under cold climate and thus gets transmitted more easily among people. Another possible factor that might contribute to the rise of $\mathrm{NoV}$ in cold season is consumption of contaminated food, such as shellfish, which is often eaten raw locally. It is through wastewater discharges that $\mathrm{NoV}$ enters the water system and leads to the contamination of shellfish in waters. Studies on the NoV contamination in oyster population indicated that oysters captured in cold seasons were more heavily contaminated by NoV than that captured during warm seasons [29].

All of the above might help in explaining the seasonal change of $\mathrm{NoV}$ infection rate in general, though the underlying mechanism for why there is an optimal condition for its replication and dissemination in human at cold seasons are still unclear. And further studies on local seafood consumption, contamination level of local seafood are needed to better understand the correlation between exposure and infection status. 


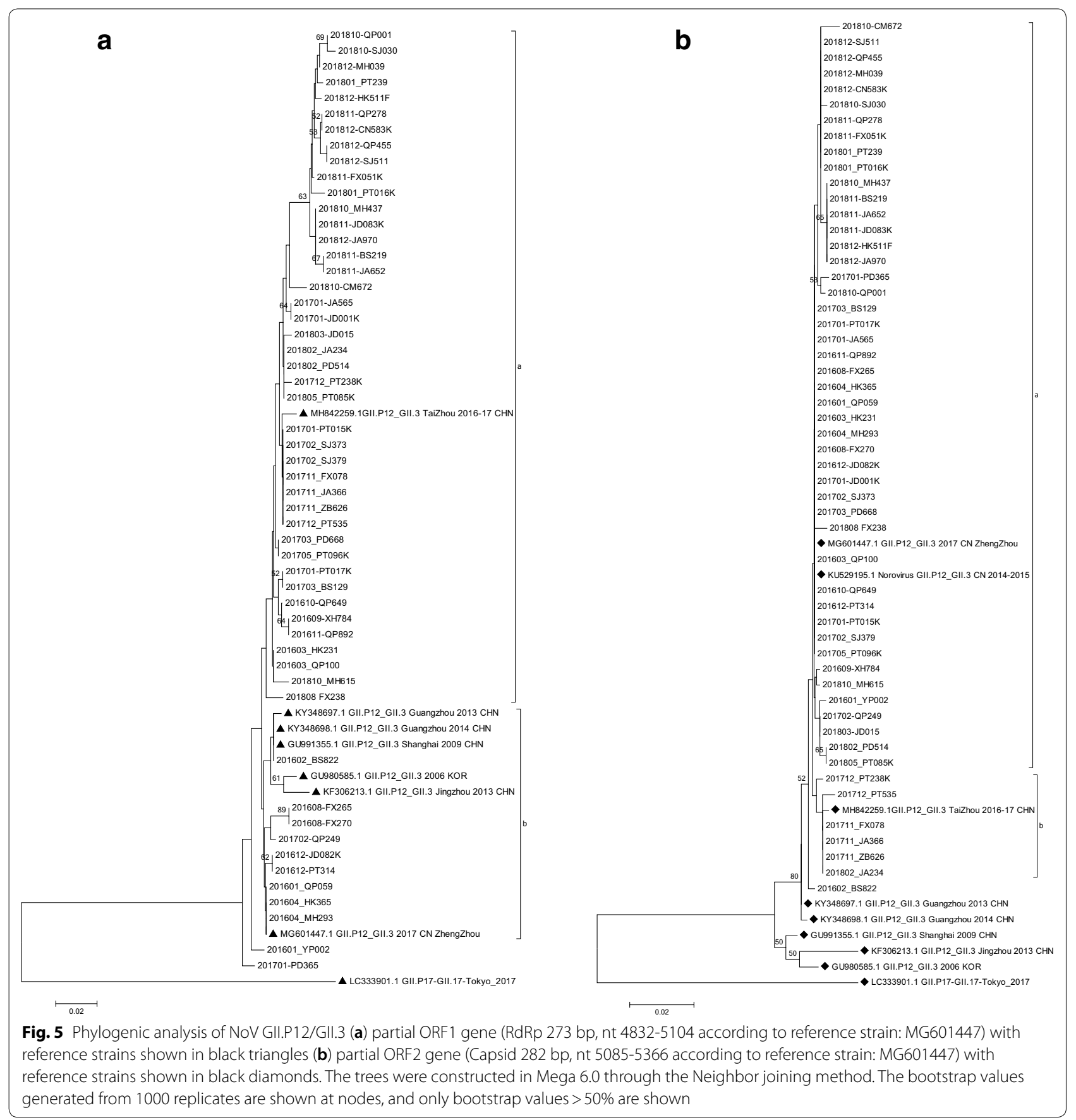

\section{NoV GIl genotypic distribution}

Majority of sequenced samples were found to be recombinant genotypes. This finding confirms that recombination is an important mechanism in generating genetic diversity in NoV. This in term results in antigenic variation, which could counteract host defense system, and even affect herd immunity in its evolution; and becomes an important factor contributing to the emergence of novel NoVs in human population [7]. This progress was reflected by the finding that despite the gradual decline of GII.Pe/GII.4 over 3 years; similar decline was not observed in GII.P17/GII.17. This is possibly owing to the fact that GII.Pe/GII.4 has a longer establishment in the population than more recently emerged GII.P17/GII.17 genotype, and it takes years for the herd immunity to fight against one particular genotype. 


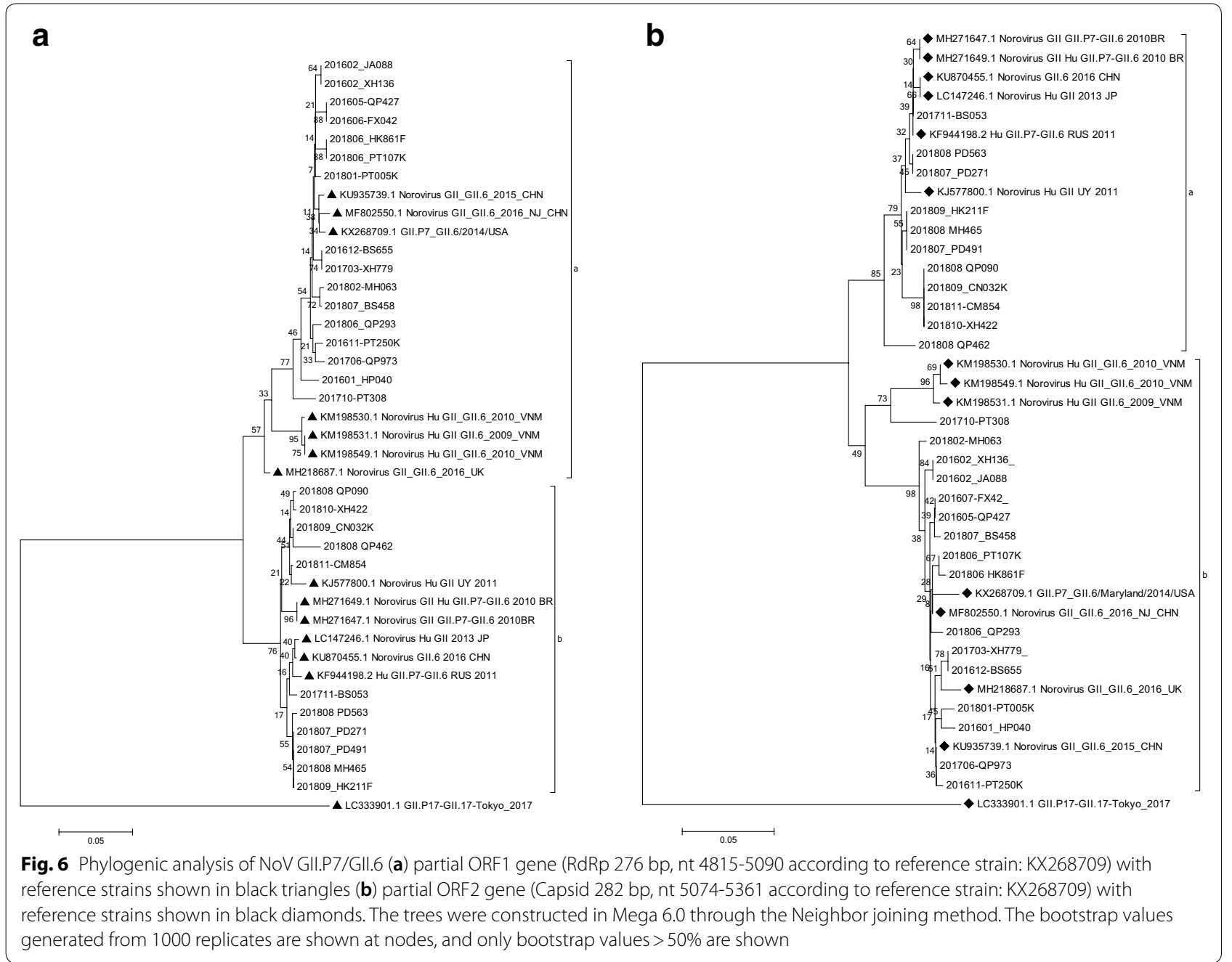

Every 2-4 years, there would emerge a new NoV strain, quite often a recombinant strain, to replace its predecessor and gains its new dominance in circulation. GII.Pe/ GII.4 Sydney strain has spread to the world and China since 2012 [3, 30, 31], and then in the winter of 2014, a new GII.P17/GII.17 strain start to emerge in Guangdong, Japan, US and other part of the world [32-34]. Another 2 years had passed before the emergence of a new recombinant GII.P16/GII.2 in the winter of 2016 throughout the world [13-15]. The prevalence of different NoV GII genotypes and the appearance of GII.P16/GII.2 recombinant strain in this study were consistent with the general evolvement of NoV GII in the rest of the world. It is also worth noting that a rise in NoV GII detection rate in 2017 coincided with the new emergence and rise of GII.P16/ GII.2 recombinant genotype.

During 2016-2017, GII.4 genotype and other nonGII.4 genotypes had displayed a bimodal seasonal alternating pattern, that is, GII.4 dominated the epidemic in autumn-winter season, whereas non-GII.4 genotypes dominated the spring-summer season. These results were consistent with the finding in Hong Kong [35] although their seasonality is different from this study probably attributed to a difference in regional climate. It was proposed that this peculiar pattern might reflect a complex virus-human immunologic interaction on individual and population levels; and like in influenza virus, the mechanism itself might be used by the virus to generate antigenic novelty $[35,36]$. More detailed research on why each NoV GII genotype had exhibited their distinct circulation pattern was still under progress, and should be considered for future study. However, in contrast to the bimodal pattern exhibited during 2016-2017, the prevalence pattern in 2018 was characterized by the coexistence of multiply genotypes. This change in prevalence pattern might be another mode in NoV evolution as the co-circulation provides a potential opportunity for gene exchange among different genotypes and might 
encourage the emergence of new strains. However further investigations are necessary for the confirmation of such hypothesis.

In addition, this study showed that the prevalence of GII.4 by the end of 2018 still remained higher than any other types, which is different from the finding in Shanghai Pudong New Area, in which the prevalence GII.P17/ GII.17 has already exceeded GII.Pe/GII.4 by the middle of 2015 [37]. This discrepancy might be due to the difference in sampling techniques and area representativeness.

\section{Less reported recombinant genotypes such as GII.P12/GII.3} and GII.P7/GII.6 that had risen recently in adult population Simultaneous presence of multiple gene clusters was observed in both GII.P12/GII.3 and GII.P7/GII.6 recombinants. This might provide opportunity for genetic exchange among different variants of the same genotype for the emergence of new strains. Studies have shown that GII.3 NoV had one of the highest detection rates in sporadic cases before the 1997-1990s, and its evolution rate was approximately $4.16 \times 10^{-3}$ base substitution/ site/year, which was almost as high as the more prevalent GII.4 [38]. But unlike GII.4, the selective evolutionary pressure of GII.3 is mainly driven by the host [38]. On the other hand, GII.P7/GII.6 cannot be ignored for this recombinant strain has been present in Asia and the world for at least 20 years, and has been frequently found in recent outbreaks, accompanied with a significant increase of sequence submission to GenBank [17, 18]. Therefore it is essential to carry on the routine monitoring of the above recombinant strains for their prevalence change. However for more insight into whether these gene variations in different clusters would indeed affect the binding of the virus to the host receptor or the infectivity of virus, longer sequence analysis covering complete genome and protein structure analysis will be required in future study.

\section{Conclusion}

While an alternating predominance of GII.4 and NonGII.4 was observed in Shanghai gastroenteritis outpatients in 2016-2017, the circulation pattern of NoV GII was less distinct in 2018 with the co-prevalence of multiple genotypes. A recent increase in detection rate in less reported recombinant genotypes such as GII.P12/GII.3 and GII.P7/GII.6 among adult population, together with the discovery of multiple gene clustering in the ORF1ORF2 junction of both genotypes calls for a continuing close monitoring on NoV GII genotypes in case of potential local outbreaks.

\section{Additional file}

Additional file 1. GenBank accession numbers of sequences for strains of interest.

\section{Acknowledgements}

We'd like to thank 27 sentinel hospitals and 16 district labs for their continuous contribution to Shanghai Comprehensive Gastroenteritis Surveillance Programme.

\section{Authors' contributions}

XK performed the experiments, conducted the analysis, and drafted and revised the manuscript. ZT helped in refine the analysis and revised the manuscript. XZ designed and supervised the study and commented on the manuscript. All authors have ensured the accuracy and integrity of this article. All authors read and approved the final manuscript.

\section{Funding}

This work was supported by National "Thirteenth Five-Year" Programme of Key Research and Development for Infectious Disease Prevention (2017ZX10103009-003). The funders had no role in study design, data collection and analysis, decision to publish, or preparation of the manuscript.

\section{Availability of data and materials}

All data involved in this study is available upon reasonable request made to the corresponding author.

\section{Ethics approval and consent to participate}

The study protocol was approved by the Ethics Review Board of Shanghai Municipal Center for Disease Control and Prevention, China. Clinical stool samples were obtained from the routine Comprehensive Gastroenteritis Surveillance Programme of Shanghai CDC. Sample collection was approved by either the patients or their parents with prior informed consent.

\section{Consent for publications}

Not applicable.

\section{Competing interests}

The authors declare that they have no competing interests.

Received: 17 April 2019 Accepted: 20 July 2019

Published online: 26 July 2019

\section{References}

1. Ahmed SM, Hall AJ, Robinson AE, et al. Global prevalence of norovirus in cases of gastroenteritis: a systematic review and meta-analysis. Lancet Infect Dis. 2014:14(8):725-30.

2. Centers for Disease Control and Prevention. Emergence of new norovirus strain Gll.4 Sydney_United States, 2012. MMWR Morb Mortal Wkly Rep. 2013;62(3):55

3. Van Beek J, Ambert-Balay K, Botteldoorn N, et al. Indications for worldwide increased norovirus activity associated with emergence of a new variant of genotype II.4, late 2012. Eurosurveillance. 2013;18(1):8-9.

4. Bartsch SM, Lopman BA, Ozawa S, et al. Global economic burden of norovirus gastroenteritis. PLoS ONE. 2016;11(4):e0151219.

5. Li J, Pan H, Xiao WJ, et al. Epidemiological and etiological surveillance study of infectious diarrhea in Shanghai in 2013-2015. Zhonghua Yu Fang Yi Xue Za Zhi. 2017;51(12):1113-7.

6. Xue $\mathrm{Y}, \mathrm{Pan} \mathrm{H}, \mathrm{Hu}$ J, et al. Epidemiology of norovirus infections among diarrhea outpatients in a diarrhea surveillance system in Shanghai, China: a cross-sectional study. BMC Infect Dis. 2015;15:183.

7. De Graaf M, Van Beek J, Koopmans MPG. Human norovirus transmission and evolution in a changing world. Nat Rev Microbiol. 2016;14:421.

8. He Y, Jin M, Chen K, et al. Gastroenteritis outbreaks associated with the emergence of the new GIl.4 Sydney norovirus variant during the epidemic of 2012/13 in Shenzhen City, China. PLOS ONE. 2016;11(11):e0165880. 
9. Qin SW, Chan TC, Cai J, et al. Genotypic and epidemiological trends of acute gastroenteritis associated with noroviruses in China from 2006 to 2016. Int J Environ Res Public Health. 2017. https://doi.org/10.3390/ijerp h14111341.

10. Ao Y, Cong X, Jin M, et al. Genetic analysis of reemerging Gll.P16-GIl.2 noroviruses in 2016-2017 in China. J Infect Dis. 2018;218(1):133-43.

11. Shen Z, Wang G, Zai SB, et al. The detection of novel norovirus Sydney strain Gll.4 in Shanghai. Chin J Virol. 2013;6:608-14.

12. Pan LF, et al. The novel norovirus genotype Gll.17 is the predominant strain in diarrheal patients in Shanghai, China. Gut Pathog. 2016:8(1):49.

13. Niendorf $S$, Jacobsen S, Faber M, et al. Steep rise in norovirus cases and emergence of a new recombinant strain Gll.P16-Gll.2, Germany, winter 2016. Eurosurveillance. 2017;22(4):30447.

14. Bidalot M, Théry L, Kaplon J, et al. Emergence of new recombinant noroviruses Gll.p16-Gll.4 and Gll.p16-GIl.2, France, winter 2016 to 2017. Eurosurveillance. 2017;22(15):30508.

15. Kwok K, Niendorf S, Lee N, et al. Increased detection of emergent recombinant norovirus GII.P16-GIl.2 strains in young adults, Hong Kong, China, 2016-2017. Emerg Infect Dis. 2017;23(11):1852-5.

16. Liu J, Li S, Wang C, et al. Genomic characterization of Gll.3 noroviruses isolated from children in Zhengzhou city, China, 2015/16. Arch Virol. 2018;163(10):2737-42.

17. Dabilla N, Almeida TNV, Franco FC, et al. Recombinant noroviruses detected in Mid-West region of Brazil in two different periods 2009-2011 and 2014-2015: atypical breakpoints of recombination and detection of distinct GII.P7-GII.6 lineages. Infect Genet Evol. 2019;68:47-53.

18. Dong $X$, Qin M, Wang Z, et al. Should we pay attention to recombinant norovirus strain GII.P7/GIl.6? J Infect Public Health. 2019;12:403-9.

19. The World Health Organization; 2014. http://www.who.int/topics/diarr hoea/en/. Accessed 18 Nov 2014.

20. Cannon JL, Barclay L, Collins NR, et al. Genetic and epidemiologic trends of norovirus outbreaks in the United States from 2013 to 2016 demonstrated emergence of novel Gll.4 recombinant viruses. J Clin Microbiol. 2017;55(7):2208-21.

21. Sequencher ${ }^{\circledR}$ DNA Sequence Analysis Software Gene Codes Corporation, Ann Arbor, Mi USA, http://www.genecodes.com. Accessed 29 Dec 2018.

22. Tamura K, Peterson D, Peterson N, et al. MEGA5: molecular evolutionary genetic analysis using maximum likelihood, evolutionary distance, and maximum parsimony methods. Mol Biol Evol. 2011;28:2731-9.

23. Yu J, Lai S, Wang X, et al. Analysis of epidemiology characteristics of norovirus among diarrheal outpatients in 27 provinces in China, 2009-2013. Zhonghua Liu Xing Bing Xue Za Zhi. 2015;36(3):199-204.

24. Zhang SX, Li L, Yin JW, et al. Emergence of human caliciviruses among diarrhea cases in southwest China. BMC Infect Dis. 2016:16(1):511.

25. Jiang $C L, X u Y$, Liu Y, et al. Analysis of rotavirus and norovirus infections among infants with diarrhea in an outpatient in Nanjing. Int J Virol. 2018;25(6):392-6.
26. Wang P, Goggins W, Chan E. A time-series study of the association of rainfall, relative humidity and ambient temperature with hospitalizations for rotavirus and norovirus infection among children in Hong Kong. Sci Total Environ. 2018;643:414-22.

27. Kauppinen A, Miettinen IT. Persistence of norovirus Gll genome in drinking water and wastewater at different temperatures. Pathogens. 2017;6(4):1.

28. Bozkurt H, D'souza DH, Davidson PM. Thermal inactivation of foodborne enteric viruses and their viral surrogates in foods. J Food Prot. 2015;78(8):1597-617.

29. Tan DM, Lyu SL, Liu W, et al. Utility of droplet digital pcr assay for quantitative detection of norovirus in shellfish, from production to consumption in Guangxi, China. Biomed Environ Sci. 2018;31(10):713-20.

30. Huo Y, Cai A, Yang H, et al. Complete nucleotide sequence of a norovirus Gll.4 genotype: evidence for the spread of the newly emerged pandemic Sydney 2012 strain to China. Virus Genes. 2014;48(2):356-60.

31. Wu F, Chen $\mathrm{H}$, Yen C, et al. Epidemiology and molecular characteristics of norovirus Gll.4 Sydney outbreaks in Taiwan, January 2012-December 2013. J Med Virol. 2015;87(9):1462-70.

32. Lu J, Sun L, Fang L, et al. Gastroenteritis outbreaks caused by norovirus GIl.17, Guangdong Province, China, 2014-2015. Emerg Infect Dis. 2015;21(7):1240-2.

33. Matsushima Y, Ishikawa M, Shimizu T, et al. Genetic analyses of Gll.17 norovirus strains in diarrheal disease outbreaks from December 2014 to March 2015 in Japan reveal a novel polymerase sequence and amino acid substitutions in the capsid region. Eurosurveillance. 2015;20(26):21173.

34. Parra Gl, Green KY. Genome of emerging norovirus GIl.17, United States, 2014. Emerg Infect Dis. 2015;21(8):1477-9.

35. Chan MC, Kwok K, Zhang L, et al. Bimodal seasonality and alternating predominance of norovirus Gll.4 and non-Gll.4, Hong Kong, China, 2014-2017(1). Emerg Infect Dis. 2018;24(4):767-9.

36. Langat P, Raghwani J, Dudas G, et al. Genome-wide evolutionary dynamics of influenza $B$ viruses on a global scale. PLoS Pathog. 2017;13(12):e1006749.

37. Pan L, Xue C, Fu H, et al. The novel norovirus genotype Gll.17 is the predominant strain in diarrheal patients in Shanghai, China. Gut pathog. 2016;8:49. https://doi.org/10.1186/s13099-016-0131-3.

38. Boon D, Mahar JE, Abente EJ, et al. Comparative evolution of GIl.3 and Gll.4 norovirus over a 31-year period. J Virol. 2011;85(17):8656-66.

\section{Publisher's Note}

Springer Nature remains neutral with regard to jurisdictional claims in published maps and institutional affiliations.
Ready to submit your research? Choose BMC and benefit from:

- fast, convenient online submission

- thorough peer review by experienced researchers in your field

- rapid publication on acceptance

- support for research data, including large and complex data types

- gold Open Access which fosters wider collaboration and increased citations

- maximum visibility for your research: over 100M website views per year

At BMC, research is always in progress.

Learn more biomedcentral.com/submissions 